\title{
Aportes al conocimiento de la biota liquénica del oasis de neblina de Alto Patache, Desierto de Atacama'
}

\author{
Reinaldo Vargas Castillo², Daniel Stanton ${ }^{3}$ y Peter R. Nelson ${ }^{4}$
}

\begin{abstract}
RESUMEN
Los denominados oasis de neblina son áreas en las zonas costeras del Desierto de Atacama donde el ingreso habitual de niebla permite el establecimiento y desarrollo de diversas poblaciones de plantas vasculares, generando verdaderos hotspots de diversidad. En estas áreas, la biota liquenológica ha sido poco explorada y representa uno de los elementos perennes más importantes que conforman la comunidad. En un estudio previo de la biota del oasis de neblina de Alto Patache se reportaron siete especies. Con el fin de mejorar este conocimiento, se analizó la riqueza de especies presentes en el oasis siguiendo dos transectos altitudinales en diferentes orientaciones del farellón. Aquí reportamos preliminarmente 77 especies de líquenes para el oasis de neblina de Alto Patache. De estas, 61 especies corresponden a nuevos registros para la región de Tarapacá, en tanto que las especies Amandinea efflorescens, Diploicia canescens, Myriospora smaragdula y Rhizocarpon simillimum corresponden a nuevos registros para el país. Asimismo, se destaca a Alto Patache como la única localidad conocida para Santessonia cervicornis, una especie endémica y en Peligro Crítico.
\end{abstract}

Palabras clave: Oasis de neblina, Desierto de Atacama, líquenes.

\begin{abstract}
Fog oases are zones along the Atacama Desert where the regular input of fog favors the development of rich communities of vascular plants, becoming biodiversity hotspots. In these areas, the lichen biota has been poorly explored and represents one of the most conspicuous elements among the perennials organisms that form the community. In a previous study of the lichen biota of the fog oasis at Alto Patache 7 species were reported. With the intent of update this information, lichen richness was assessed following 2 altitudinal transects at different aspects of the coastal bluff. Here we present preliminary data indicating the presence of 77 species. Of these, 61 species are new records for the Tarapacá Region, and the species Amandinea efflorescens, Diploicia canescens, Myriospora smaragdula and Rhizocarpon simillimum are new records for the Chilean lichen flora. Alto Patache is also acknowledged as the sole locality for Santessonia cervicornis, an endemic Critically Endangered species.
\end{abstract}

Key words: Fog oasis, Atacama Desert, lichens.

Los autores agradecen al equipo del Centro UC Desierto de Atacama (CDA) de la Pontificia Universidad Católica de Chile por el apoyo logístico y las facilidades entregadas para realizar esta investigación. Al Dr. Horacio Larraín y a la profesora Pilar Cereceda, quienes fueron los primeros en mostrarnos la diversidad de líquenes de Alto Patache. Este estudio fue financiado con el apoyo del proyecto National Geographic Society Committee on Research and Exploration (\#9578-14). Artículo recibido el 20 de enero de 2017, aceptado el 11 de septiembre de 2017 y corregido el 31 de octubre de 2017

2 Herbario Federico Johow / Departamento de Biología, Universidad Metropolitana de Ciencias de la Educación (Chile). E-mail: reinaldovargas@gmail.com

Department of Ecology, Evolution and Behavior, University of Minnesota-Twin Cities (USA). E-mail: stan0477@umn.edu

4. Natural and Behavioral Sciences, University of Maine-Fort Kent (USA) E-mail: peter.nelson@maine.edu 
Los oasis de neblina representan de manera habitual un hotspot de biodiversidad, particularmente de plantas vasculares en el habitualmente hiperárido desierto costero del norte de Chile. En estos ecosistemas, la mayor parte del agua ingresa por efecto de la condensación de la neblina costera, y se restringen a pequeñas áreas donde la topografía de la Cordillera de la Costa favorece la captura del flujo aéreo marino.

Aunque pequeños en superficie, estos ambientes usualmente poseen una alta diversidad de plantas y animales, muchos de ellos adaptados de manera única a estas condiciones. Como resultado de su riqueza biológica estos sitios poseen, además, una gran importancia arqueológica (Larraín et al., 2004).

Los oasis de neblina tienen una larga historia de exploración botánica (Phillippi, 1884, 1885), no obstante, en parte a las dificultades en su acceso y a la fuerte importancia dada a los infrecuentes eventos de lluvia, aún siguen siendo pobremente exploradas florísticamente. Esfuerzos recientes por establecer y mapear la vegetación de estas "lomas" ha ampliado altamente nuestro conocimiento (Pinto \& Luebert, 2009; Schulz, 2009), del modo como lo han hecho expediciones posterior a eventos El Niño - Oscilación del Sur (ENOS) (Muñoz-Schick et al., 2001). Varios grupos icónicos han sido objeto de documentación y colección más detallada, como cactaceae (Lembcke \& Weisser, 1979; Zuloaga et al., 2008), bromelias (Rundel \& Dillon, 1998) y el género Nolana (Dillon et al., 2007; Tu et al., 2008; Ossa et al., 2013; Cabrera et al., 2015).

A pesar del aumento de la atención hacia la vegetación de lomas, poca atención se le ha dado a uno de los componentes dominantes de las comunidades bióticas del Desierto de Atacama, los líquenes. Estos parecen estar particularmente bien adaptados a las condiciones de niebla en el desierto, con una fisiología capaz de soportar repetidos ciclos de hidratación y secado (Rundel, 1978; Rundel, 1982; Lange \& Redón, 1982; Lange et al., 2006).

El estudio de la diversidad de líquenes en Chile ha sido escaso y de baja profundidad. Este se ha basado principalmente en la realización de listados florísticos de diferentes zonas de interés del extremo norte y sur del país, o en zonas de particular interés botánico (Galloway \& Marticorena, 1991; Galloway, 1998; Quilhot et al., 1998). Entre estos destacan zonas del Altiplano de las regiones de Arica y Parinacota, y Tarapacá (Galloway, 1998); oasis de neblina en el desierto costero y la zona costera del centro de Chile (Follmann, 1965, 1968, 1995); el bosque templado lluvioso o valdiviano (Galloway, 1995); zonas precordilleranas de Chile central (Pereira \& San Martín, 1998, Pereira et al., 1999, 2002, 2006, 2014, 2016; Redón \& Walkowiak, 1978); de las zonas subantárticas

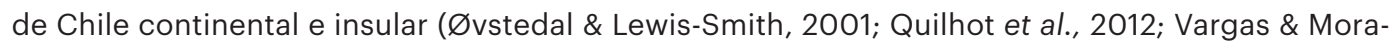
no, 2014); y el Archipiélago de Juan Fernández (Zahlbruckner, 1924; Redón \& Quilhot, 1977).

Para Chile, se ha mencionado la presencia de aproximadamente 1.400 especies de hongos liquenizados (Galloway \& Quilhot, 1998). Esta aproximación está basada en revisiones bibliográficas y podrían representar sólo un bajo grado de conocimiento del grupo en el país a la fecha de la publicación del listado (Galloway \& Quilhot, 1998), y podrían considerar valores cercanos a las 2.500 especies (Follmann, 1995). Por otro lado, y de manera general, se adolece de estudios ecológicos sobre estructura poblacional y comunitaria en hongos liquenizados, con algunas excepciones en especies del bosque valdiviano (Lücking et al., 2003; Rundel, 1980; Redón, 1973), en su utilización como organismos modelo en estudios ecológicos (Armesto \& Contreras, 1981), y 
en estudios de la selectividad de micobiontes por sus fotobiontes en zonas del desierto costero de la región de Tarapacá (Vargas \& Beck, 2012).

La biota liquenizada del norte de Chile fue estudiada parcialmente por Follmann en la segunda mitad del siglo XX (Follmann, 1964, 1965, 1966, 1967, 1994, 1995). Estos estudios contribuyeron enormemente al conocimiento de la diversidad de líquenes en Chile, pero se ha hecho necesario el actualizar esta información a la luz de cambios nomenclaturales (James et al., 2006, Schoch et al., 2009; Jaklitsch et al., 2016), cambios en los rangos distribucionales de las especies (Follmann 1995), y completar los vacíos en zonas geográficas no exploradas. Esta última es la intención de este trabajo.

El oasis de neblina de Alto Patache, traído a la luz como resultado del evento ENOS de 19971998, se ha situado como un significativo hotspot de diversidad de plantas vasculares en el norte de Chile (Muñoz-Schick et al., 2001). Las condiciones microclimáticas que favorecen la alta diversidad de plantas vasculares (nieblas frecuentes y densas) se espera que favorezcan de igual manera la diversidad liquénica. En la zona se ha reportado la presencia de sólo siete especies de líquenes en el contexto de una exploración botánica de la flora vascular presente (Muñoz-Schick et al., 2001), y dado que el muestreo no estaba orientado a este grupo, se vuelve indudable el considerar que la diversidad actual está fuertemente subestimada. Una indicación a la diversidad real puede ser observada en el único trabajo publicado orientado al estudio de líquenes en Alto Patache: el estudio de Vargas \& Beck (2012) en seis representantes del género Caloplaca, un género que no había sido mencionado en el estudio previo.

Dado el potencial de una muy alta diversidad de líquenes en Alto Patache, basado tanto en observaciones personales previas como en los estudios mencionados, establecimos un estudio estratificado altitudinalmente de las comunidades de líquenes presentes en Alto Patache, combinando conocimiento experto con muestreos sistemáticos diseñados para proveer información acabada sobre esta comunidad de líquenes de desiertos de neblina. En este trabajo reportamos los resultados preliminares de este estudio entregando un listado de especies presentes en el oasis de neblina de Alto Patache.

\section{Materiales y Métodos}

Los muestreos fueron llevados a cabo en distintos puntos del farellón costero del oasis de neblina de Alto Patache, en los alrededores de la Estación Atacama UC Oasis Niebla Alto Patache en colaboración con el Centro del Desierto de Atacama de la Pontificia Universidad Católica de Chile (Figura $N^{\circ} 1$ ). Dado que diferentes especies de líquenes pueden estar adaptadas a diferentes condiciones microambientales, se hace necesario el muestrear a lo largo del rango altitudinal del oasis de neblina para capturar de mejor manera la diversidad presente. De este modo, los muestreos se realizaron a lo largo de dos gradientes altitudinales en el farellón costero con diferente exposición, considerando todos los sustratos disponibles para el establecimiento de líquenes.

Los gradientes se iniciaron en el límite inferior de la presencia de líquenes hasta la parte superior del farellón. El Transecto A consideraba desde los 450 m s.n.m. $\left(20^{\circ} 49,966^{\prime} \mathrm{S}-70^{\circ} 9,158^{\prime} \mathrm{O}\right)$ hasta los 850 m s.n.m. $\left(20^{\circ} 49,604^{\prime} \mathrm{S}-70^{\circ} 8,903^{\prime} \mathrm{O}\right)$ y fue muestreado en intervalos de $50 \mathrm{~m}$ de 
alto en marzo 2015 utilizando un sistema de cuadrantes y transectos ortogonales al gradiente altitudinal. El Transecto B consideraba desde los 300 m s.n.m. ( $\left.20^{\circ} 49,889^{\prime} \mathrm{S}-70^{\circ} 9,899^{\prime} \mathrm{O}\right)$ hasta los 800 m s.n.m. ( $\left.20^{\circ} 49,514^{\prime} \mathrm{S}-70^{\circ} 9,519^{\prime} \mathrm{O}\right)$ y fue muestreado en intervalos de $100 \mathrm{~m}$ de alto en Enero 2016 en forma semejante al Transecto A. Asimismo, se incluyeron puntos aleatorios en el sector denominado como Pampa Bugueño ( $20^{\circ} 49,649^{\prime} \mathrm{S}-70^{\circ} 8,531^{\prime} \mathrm{O}$ ) (no incluido en la Figura No 1). Estos muestreos fueron realizados como parte del levantamiento de información asociados a un proyecto de evaluación de forma y función de comunidades liquénicas en un oasis de neblina, siendo estos los primeros resultados publicados de esta evaluación.

Figura No 1

Ubicación de los transectos altitudinales en el oasis de neblina de Alto Patache

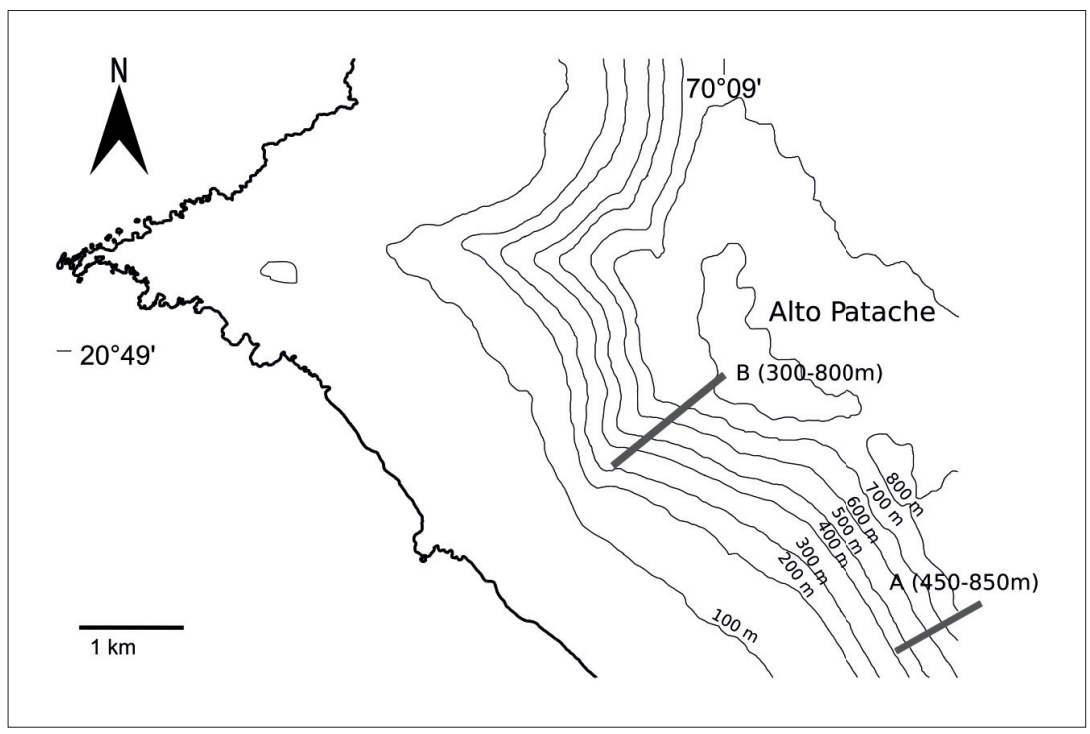

Fuente: Elaboración propia.

En cada elevación del gradiente altitudinal se establecieron transectos de 50 metros de largo perpendiculares al farellón. En ellos se colectaron los líquenes en cuadrantes de $30 \times 30 \mathrm{~cm}$ separados en intervalos de cinco metros a lo largo de los transectos. Este muestreo estratificado permite iguales esfuerzos de colecta, así como la generación de información de grano fino para un futuro estudio de análisis comunitario de líquenes.

Observaciones anatómicas y morfológicas se llevaron a cabo con microscopía óptica de los materiales colectados. Secciones de apotecios fueron estudiadas en agua y $\mathrm{KOH}$ al $10 \%$. Reacciones amiloides en el himenio, y observaciones del asca, fueron testeadas con solución lugol, luego de ser tratadas con $\mathrm{KOH}$ al 10\%. El material fue observado en una lupa estereoscópica Leica S6D y un microscopio Leica DM500 (hasta 1000x). Los materiales colectados se encuentran almacenados en el Herbario Federico Johow de la Universidad Metropolitana de Ciencias de la Educación, Santiago de Chile. La organización sistemática de las especies se siguió el esquema de Hibbett et al. (2007), Lumbsch \& Huhndorf (2010), Jaklitsch et al. (2016) y Lücking et al. (2017). 
Para las especies registradas se indica la distribución biogeográfica mundial en función del grado de disponibilidad de la información. Para líquenes se sigue la propuesta de Galloway (2008), que se basa parcialmente en las distribuciones florísticas propuestas por Takhtajan (1986), y reevaluadas para hongos liquenizados (Feuerer \& Hawksworth, 2007; Feuerer \& Höhner, 2017).

\section{Riqueza de líquenes en el oasis de neblina de Alto Patache}

Los resultados preliminares indican la presencia de 77 especies de líquenes, pertenecientes a 1 Phyllum, 3 Clases, 11 Órdenes (con 2 posiciones inciertas), 18 Familias (1 posición incierta) y 45 géneros. De las entidades determinadas, 70 han sido identificados a nivel de especies, en tanto que los 7 restantes han sido asignados a nivel de especie, pero con incertidumbres en sus identidades. De los 77 taxa, 4 corresponden a nuevos registros para el país, en tanto que 61 corresponden a nuevos registros para la región de Tarapacá. En el Cuadro No 1 se presenta el listado de especies junto con la distribución de sus presencias en el gradiente altitudinal, indicando los registros nuevos para la Región y aquellos que son nuevos para el país.

\section{Cuadro No 1}

Listado de especies y distribución a lo largo de los gradientes altitudinales estudiados. Se indica la distribución biogeográfica de las especies sensu Galloway (2008)

\begin{tabular}{|c|c|c|c|c|c|c|c|c|c|c|}
\hline Familia & Especie & $\begin{array}{l}\dot{\xi} \\
\dot{\zeta} \\
\dot{\omega} \\
\varepsilon \\
0 \\
O \\
O\end{array}$ & 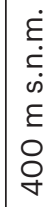 & 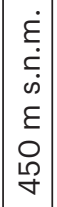 & 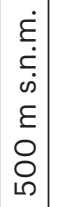 & 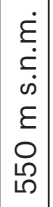 & 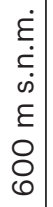 & 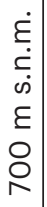 & 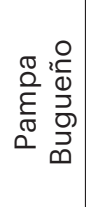 & $\begin{array}{l}\text { Distribución } \\
\text { biogeográfica }\end{array}$ \\
\hline Acarosporaceae & $\begin{array}{l}\text { Acarospora rouxii K. Knudsen, } \\
\text { Elix \& Reeb * }\end{array}$ & $x$ & & & & $x$ & & $x$ & & Neoaustral \\
\hline Caliciaceae & $\begin{array}{l}\text { Amandinea efflorescens } \\
\text { (Müll. Arg.) Marbach \# }\end{array}$ & & $x$ & & & & $\mathrm{x}$ & & & Pantropical \\
\hline Arthoniaceae & $\begin{array}{l}\text { Arthonia atacamensis } \\
\text { Follmann * }\end{array}$ & & & $x$ & & & & $\mathrm{x}$ & & Endémica \\
\hline Arthoniaceae & $\begin{array}{l}\text { Arthonia australis (Zahlbr.) } \\
\text { C.W. Dodge * }\end{array}$ & & & & & & $\mathrm{x}$ & $x$ & & Endémica \\
\hline Arthoniaceae & $\begin{array}{l}\text { Arthonia follmannii C.W. } \\
\text { Dodge * }\end{array}$ & & & $x$ & & $x$ & & $x$ & & Endémica \\
\hline Roccellaceae & $\begin{array}{l}\text { Arthoniactis chilena C.W. } \\
\text { Dodge* }\end{array}$ & & $x$ & $x$ & & & & & & Endémica \\
\hline Arthopyreniaceae & $\begin{array}{l}\text { Arthopyrenia planorbis (Ach.) } \\
\text { Müll. Arg. * }\end{array}$ & & & $x$ & $x$ & $x$ & & $x$ & & Pantropical \\
\hline Arthoniaceae & $\begin{array}{l}\text { Arthothelium chilense C.W. } \\
\text { Dodge * }\end{array}$ & & $x$ & & $x$ & $x$ & & $\mathrm{x}$ & & Endémica \\
\hline Arthoniaceae & $\begin{array}{l}\text { Arthothelium halophilum } \\
\text { Follmann * }\end{array}$ & $x$ & $x$ & $x$ & $x$ & $x$ & & $x$ & & Endémica \\
\hline Megasporaceae & $\begin{array}{l}\text { Aspicilia aff. phaea Owe- } \\
\text { Larss. \& A. Nordin * }\end{array}$ & & & & & $x$ & & $x$ & & Bipolar \\
\hline
\end{tabular}


Continuación Cuadro No 1

\begin{tabular}{|c|c|c|c|c|c|c|c|c|c|c|}
\hline Familia & Especie & 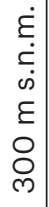 & 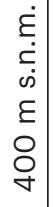 & 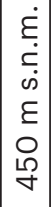 & $\mid \begin{array}{c}\dot{\varepsilon} \\
\dot{\dot{L}} \\
\dot{\omega} \\
\varepsilon \\
\subseteq \\
\bigcirc \\
\llcorner\end{array}$ & 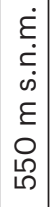 & $\begin{array}{c}\dot{\varepsilon} \\
\dot{L} \\
\dot{\omega} \\
\varepsilon \\
0 \\
\bigcirc \\
\varnothing\end{array}$ & 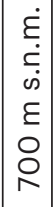 & 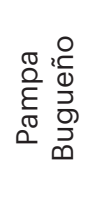 & $\begin{array}{l}\text { Distribución } \\
\text { biogeográfica }\end{array}$ \\
\hline Aspidotheliaceae & $\begin{array}{l}\text { Aspidopyrenis cf. insignis } \\
\text { (Vain.) Clem. \& Shear * }\end{array}$ & $x$ & & $x$ & & & & & & Neoaustral \\
\hline Caliciaceae & $\begin{array}{l}\text { Buellia albula (Nyl.) Müll. Arg. } \\
\text { * }\end{array}$ & $x$ & & & $x$ & & & & & Austral \\
\hline Caliciaceae & $\begin{array}{l}\text { Buellia coquimbensis C.W. } \\
\text { Dodge * }\end{array}$ & & & & $x$ & $x$ & & & & Endémica \\
\hline Caliciaceae & Buellia jorgensis Zahlbr. * & $x$ & & $x$ & $\mathrm{x}$ & $x$ & $x$ & $\mathrm{x}$ & & Endémica \\
\hline Caliciaceae & Buellia pirionii B. de Lesd. * & $x$ & & & & & & $\mathrm{x}$ & & Endémica \\
\hline Caliciaceae & $\begin{array}{l}\text { Buellia subdisciformis } \\
\text { (Leight.) Jatta * }\end{array}$ & & & & $x$ & $\mathrm{x}$ & $x$ & & & Cosmopolita \\
\hline \begin{tabular}{|l} 
Caliciaceae \\
\end{tabular} & Buellia tristicolor Zahlbr. * & & & & $x$ & $x$ & & $\mathrm{x}$ & & Neoaustral \\
\hline Chrysothricaceae & $\begin{array}{l}\text { Byssocaulon cf. niveum Mont. } \\
\text { * }\end{array}$ & & $x$ & & $x$ & & & & & Endémica \\
\hline Teloschistaceae & $\begin{array}{l}\text { Caloplaca jorgensis C.W. } \\
\text { Dodge* }\end{array}$ & & $x$ & $x$ & & & & & & Endémica \\
\hline Teloschistaceae & Caloplaca rubina Zahlbr. * & & & & $x$ & $x$ & & $x$ & & Endémica \\
\hline Teloschistaceae & $\begin{array}{l}\text { Caloplaca subherbidella } \\
\text { (Räsänen) C.W. Dodge * }\end{array}$ & & & $x$ & $x$ & & $x$ & & & Endémica \\
\hline Candelariaceae & $\begin{array}{l}\text { Candelariella terrigena } \\
\text { Räsänen * }\end{array}$ & & & $x$ & & & $x$ & $x$ & & Cosmopolita \\
\hline Roccellaceae & Chiodecton follmannii Riedl * & & & & & $x$ & & & & Endémica \\
\hline Chrysothricaceae & $\begin{array}{l}\text { Chrysothrix granulosa G. } \\
\text { Thor * }\end{array}$ & & & & & & & $x$ & & Austral \\
\hline Chrysothricaceae & $\begin{array}{l}\text { Chrysothrix pavonii (Fr.) J.R. } \\
\text { Laundon }\end{array}$ & & & $x$ & & & & $x$ & & Neoaustral \\
\hline Graphidaceae & $\begin{array}{l}\text { Diorygma hololeucum (Mont. } \\
\text { \& Bosch) Kalb, Staiger \& Elix * }\end{array}$ & & & $x$ & & & & $x$ & & Pantropical \\
\hline Caliciaceae & $\begin{array}{l}\text { Diploicia canescens (Dicks.) } \\
\text { A. Massal. \# } \\
\end{array}$ & & & & & & $x$ & & & Bipolar \\
\hline Roccellaceae & $\begin{array}{l}\text { Enterographa atacamensis } \\
\text { C.W. Dodge * }\end{array}$ & & & & $x$ & & & $x$ & & Endémica \\
\hline Roccellaceae & $\begin{array}{l}\text { Enterographa cf. albinea } \\
\text { (Nyl.) C.W. Dodge * }\end{array}$ & & & & $x$ & $x$ & & $x$ & & Endémica \\
\hline Roccellaceae & $\begin{array}{l}\text { Enterographa follmannii C.W. } \\
\text { Dodge * }\end{array}$ & & & & & $x$ & $x$ & & & Endémica \\
\hline Teloschistaceae & $\begin{array}{l}\text { Follmannia orthoclada } \\
\text { (Zahlbr.) Frödén, Arup \& } \\
\text { Søchting }\end{array}$ & $x$ & $x$ & & $x$ & & $x$ & $x$ & & Endémica \\
\hline Roccellaceae & $\begin{array}{l}\text { Follmanniella scutellata Peine } \\
\text { \& B. Werner * }\end{array}$ & & $\mathrm{x}$ & & & & $x$ & & & Endémica \\
\hline Physciaceae & $\begin{array}{l}\text { Heterodermia galactophylla } \\
\text { (Tuck.) Culb. * }\end{array}$ & & & & & $\mathrm{x}$ & & $\mathrm{x}$ & & Pantropical \\
\hline Physciaceae & $\begin{array}{l}\text { Heterodermia leucomelos (L.) } \\
\text { Poelt * }\end{array}$ & & & & & $x$ & & & & Cosmopolita \\
\hline
\end{tabular}


Continuación Cuadro No 1

\begin{tabular}{|c|c|c|c|c|c|c|c|c|c|c|}
\hline Familia & Especie & 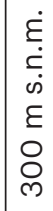 & 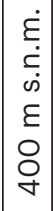 & 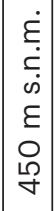 & 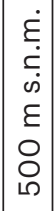 & $\begin{array}{c}\dot{\xi} \\
\dot{C} \\
\dot{\omega} \\
\varepsilon \\
0 \\
0 \\
\llcorner \\
\llcorner\end{array}$ & 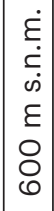 & 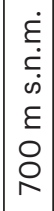 & 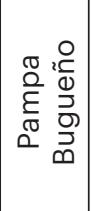 & $\begin{array}{l}\text { Distribución } \\
\text { biogeográfica }\end{array}$ \\
\hline Physciaceae & $\begin{array}{l}\text { Heterodermia multiciliata } \\
\text { (Kurok.) Trass * }\end{array}$ & & & $x$ & $x$ & & $x$ & $x$ & & Neoaustral \\
\hline Physciaceae & $\begin{array}{l}\text { Hyperphyscia cf. syncolla } \\
\text { (Tuck. ex Nyl.) Kalb * }\end{array}$ & & & & $x$ & & & $x$ & & Pantropical \\
\hline Roccellaceae & Ingaderia pulcherrima Darb. * & & & & & & $x$ & $\mathrm{x}$ & & Endémica \\
\hline Roccellaceae & $\begin{array}{l}\text { Lecanographa azurea } \\
\text { Follmann }\end{array}$ & & & & $x$ & & $x$ & $x$ & & Endémica \\
\hline Lecanoraceae & $\begin{array}{l}\text { Lecanora coquimbensis } \\
\text { Zahlbr. * }\end{array}$ & & & & & & $\mathrm{x}$ & $x$ & & Endémica \\
\hline Acarosporaceae & $\begin{array}{l}\text { Myriospora smaragdula } \\
\text { (Wahlenb.) Nägeli ex Uloth \# }\end{array}$ & & & & $x$ & & $x$ & $x$ & & Bipolar \\
\hline Ramalinaceae & $\begin{array}{l}\text { Niebla ceruchis (Ach.) Rundel } \\
\& \text { Bowler * }\end{array}$ & & & & & $x$ & $x$ & $x$ & & Bipolar \\
\hline Ramalinaceae & Niebla granulans Sipman * & & & & & & $\mathrm{x}$ & $\mathrm{x}$ & & Endémica \\
\hline Ramalinaceae & Niebla nashii Sipman * & & & & & $\mathrm{x}$ & & $\mathrm{x}$ & & Endémica \\
\hline Ramalinaceae & $\begin{array}{l}\text { Niebla tigrina (Follmann) } \\
\text { Rundel \& Bowler * }\end{array}$ & & & & & $x$ & & $x$ & & Endémica \\
\hline Roccellaceae & $\begin{array}{l}\text { Opegrapha atacamensis C.W. } \\
\text { Dodge * }\end{array}$ & & & & $x$ & & $x$ & $x$ & & Endémica \\
\hline Roccellaceae & Pentagenella fragillima Darb. * & & & & & $x$ & & $x$ & & Endémica \\
\hline Roccellaceae & $\begin{array}{l}\text { Pentagenella gracillima } \\
\text { (Kremp.) Ertz \& Tehler * }\end{array}$ & & & & & & $x$ & $x$ & & Endémica \\
\hline Physciaceae & $\begin{array}{l}\text { Physcia adscendens } \mathrm{H} \text {. } \\
\text { Olivier }\end{array}$ & & & & & $x$ & $x$ & $x$ & & Cosmopolita \\
\hline Physciaceae & Physcia cf. tenuis Moberg * & & & & $x$ & & $x$ & $x$ & & Neoaustral \\
\hline Candelariaceae & $\begin{array}{l}\text { Placomaronea kaernefeltii M. } \\
\text { Westb., Frödén \& Wedin }\end{array}$ & & & & $x$ & & $x$ & & & Endémica \\
\hline Teloschistaceae & $\begin{array}{l}\text { Polycauliona ascendens } \\
\text { (S.Y. Kondr.) Frödén, Arup \& } \\
\text { Søchting * }\end{array}$ & & & $x$ & & $x$ & $x$ & $x$ & & Endémica \\
\hline Teloschistaceae & $\begin{array}{l}\text { Polycauliona kaernefeltii (S. } \\
\text { Y. Kondr., D. J. Galloway \& } \\
\text { T. Goward) Frödén, Arup \& } \\
\text { Søchting * }\end{array}$ & & & & & & $x$ & $x$ & & Endémica \\
\hline Porinaceae & $\begin{array}{l}\text { Porina cf. chilena C.W. Dodge } \\
\text { * }\end{array}$ & & & & & $x$ & & & & Endémica \\
\hline Roccellaceae & $\begin{array}{l}\text { Protoroccella minima (R. } \\
\text { Sant.) Follmann ex Follmann }\end{array}$ & & & & & $x$ & $x$ & $x$ & & Endémica \\
\hline Ramalinaceae & $\begin{array}{l}\text { Ramalina cf. chilena (Nyl.) } \\
\text { Kashiw. * }\end{array}$ & & & & $x$ & & & $x$ & & Endémica \\
\hline Ramalinaceae & Ramalina peruviana Ach. ${ }^{*}$ & & & & & & $\mathrm{x}$ & $\mathrm{x}$ & & Pantropical \\
\hline Physciaceae & Redonia chilena C.W. Dodge * & & & $x$ & $x$ & & $x$ & & & Endémica \\
\hline Graphidaceae & $\begin{array}{l}\text { Redonographa chilensis } \\
\text { (Zahlbr.) Lücking \& Tehler * }\end{array}$ & & & & & $x$ & $x$ & $x$ & & Endémica \\
\hline
\end{tabular}




\begin{tabular}{|c|c|c|c|c|c|c|c|c|c|c|}
\hline Familia & Especie & 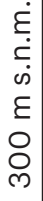 & $\left|\begin{array}{c}\dot{E} \\
\dot{C} \\
\dot{\rho} \\
E \\
0 \\
O \\
\dot{\sigma}\end{array}\right|$ & \begin{tabular}{|c|c}
$\dot{\varepsilon}$ \\
$\dot{C}$ \\
$\dot{\omega}$ \\
$\varepsilon$ \\
$\varepsilon$ \\
0 \\
$\stackrel{\overbrace{}}{\square}$ \\
\end{tabular} & 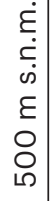 & 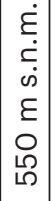 & $\begin{array}{l}\dot{\varepsilon} \\
\dot{\epsilon} \\
\dot{D} \\
\varepsilon \\
\varepsilon \\
0 \\
\bigcirc \\
0\end{array}$ & $\begin{array}{l}\dot{E} \\
\dot{C} \\
\dot{D} \\
\varepsilon \\
\qquad \\
\bigcirc \\
\bigcirc\end{array}$ & 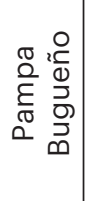 & $\begin{array}{l}\text { Distribución } \\
\text { biogeográfica }\end{array}$ \\
\hline Graphidaceae & $\begin{array}{l}\text { Redonographa saxiseda } \\
\text { (Zahlbr.) Lücking \& Tehler * }\end{array}$ & & & & & & $x$ & $x$ & & Endémica \\
\hline Rhizocarpaceae & $\begin{array}{l}\text { Rhizocarpon simillimum } \\
\text { (Anzi) Lettau \# }\end{array}$ & & & $x$ & $x$ & $x$ & $x$ & $x$ & & Bipolar \\
\hline Rhizocarpaceae & $\begin{array}{l}\text { Rhizocarpon superficiale } \\
\text { (Schaer.) Malme* }\end{array}$ & & & & $x$ & & $x$ & $x$ & & Cosmopolita \\
\hline Physciaceae & $\begin{array}{l}\text { Rinodina intrusa (Nyl.) Malme } \\
\star\end{array}$ & & & $x$ & $x$ & & & $x$ & & Austral \\
\hline Roccellaceae & $\begin{array}{l}\text { Roccellina accedens (Nyl.) } \\
\text { Tehler * }\end{array}$ & & & & & $x$ & $x$ & $x$ & & Austral \\
\hline Roccellaceae & $\begin{array}{l}\text { Roccellina cerebriformis } \\
\text { (Mont.) Tehler }\end{array}$ & & & & $x$ & $x$ & $x$ & $x$ & & Endémica \\
\hline Roccellaceae & Roccellina chalybea Tehler * & & & & & $x$ & $\mathrm{x}$ & & & Endémica \\
\hline Roccellaceae & $\begin{array}{l}\text { Roccellina limitata (Nyl.) } \\
\text { Tehler }\end{array}$ & & & & & $\mathrm{x}$ & & $\mathrm{x}$ & & Austral \\
\hline Roccellaceae & Roccellina luteola Follmann * & & & & $x$ & & & $\mathrm{x}$ & & Endémica \\
\hline Roccellaceae & $\begin{array}{l}\text { Roccellina mahuiana } \\
\text { (Follmann) Tehler }\end{array}$ & & & & & $x$ & & $x$ & & Endémica \\
\hline Roccellaceae & Roccellina nigricans Tehler * & & & & & & $\mathrm{x}$ & $\mathrm{x}$ & & Endémica \\
\hline Roccellaceae & Roccellina terrestris Tehler * & & & & & $x$ & $\mathrm{x}$ & $\mathrm{x}$ & & Endémica \\
\hline Incertae sedis & $\begin{array}{l}\text { Roccellinastrum } \\
\text { spongoideum Follmann }\end{array}$ & & & & & & & $x$ & & Endémica \\
\hline Caliciaceae & $\begin{array}{l}\text { Santessonia cervicornis } \\
\text { (Follmann) Follmann }\end{array}$ & & & & & & & & $x$ & Endémica \\
\hline Roccellaceae & $\begin{array}{l}\text { Schismatomma atacamense } \\
\text { C.W. Dodge * }\end{array}$ & & & & & & $x$ & $\mathrm{x}$ & & Endémica \\
\hline Roccellaceae & $\begin{array}{l}\text { Sclerophyton cerei (Schiman- } \\
\text { Czeika) Sipman * }\end{array}$ & & & & & $x$ & & $x$ & & Endémica \\
\hline Roccellaceae & $\begin{array}{l}\text { Sclerophyton klementii } \\
\text { (Follmann) Sipman * }\end{array}$ & & & & & & $x$ & $\mathrm{x}$ & & Endémica \\
\hline Parmeliaceae & Usnea rubicunda Stirt. & & & & & & $\mathrm{x}$ & $\mathrm{x}$ & & Cosmopolita \\
\hline Roccellaceae & $\begin{array}{l}\text { Vigneronia cf. robustula (Nyl.) } \\
\text { Ertz \& Tehler * }\end{array}$ & & & & & $x$ & & $\mathrm{x}$ & & Endémica \\
\hline
\end{tabular}

\# indica un nuevo registro para la el país

* indica un nuevo registro para la región de Tarapacá

Fuente: Elaboración propia.

\section{Riqueza de especies en el contexto del desierto costero de Chile}

Si bien el listado de especies identificadas que se presenta solo considera una fracción del total de especies presentes a lo largo de los transectos altitudinales, este indica una alta diversidad 
similar a la presente en otros oasis de neblina, como Cerro Moreno, Paposo y Fray Jorge - Altos de Talinay (Follmann, 1968, 1995; Follmann \& Redón, 1972).

Follmann (1968) indicaba la presencia de aproximadamente 160 especies en el oasis de neblina de Cerro Moreno, siendo una de las revisiones más completas de un área geográfica determinada del norte de Chile a la fecha. De estas, 144 son especies aún reconocidas taxonómicamente, de las que 81 ( 57\%) corresponden a especies endémicas para las zonas costeras del Desierto de Atacama. Para Alto Patache, destaca que el 64\% (49 especies) corresponden a taxa endémicos para el territorio nacional, y particularmente para el desierto costero del norte de Chile. Estas diferencias en riqueza y diversidad destacan aún más si se considera la reducida flora vascular perenne presente en Alto Patache, la que al funcionar como potencial sustrato para diferentes de líquenes, podría dar cuenta de la diferencia en riqueza y diversidad observada entre Cerro Moreno y Alto Patache (Follmann, 1995).

Por otro lado, la zona considerada por los diferentes oasis está dominada por un bioclima de tipo tropical desértico a hiperdesértico (Luebert \& Pliscoff, 2006; Moreira-Muñoz, 2011), caracterizado por escasas precipitaciones líquidas usualmente asociadas a eventos ENOS con un ingreso habitual de neblina o "camanchaca". Esta característica es compartida con las zonas costeras del sur de Perú, por lo que la potencial extensión en la distribución de las especies presentes en el área de estudio estaría determinada por la continuidad de este bioclima.

Las familias Roccellaceae y Caliciaceae son las más ricas en especies en los transectos estudiados. Sólo la familia Roccellaceae considera 24 especies, equivalente a $30 \%$ de las especies presentes en Alto Patache identificadas a la fecha, y correspondiendo a cerca del $40 \%$ del total de especies de la familia presentes en el país, convirtiéndose así en un hotspot de diversidad de la familia Roccellaceae (Follmann, 2001; Tehler et al., 2009). Por su parte, la familia Caliciaceae está representada por 9 especies, eminentemente del género Buellia, con 6 representantes identificados. En la actualidad no existen tratamientos taxonómicos de este género para Sudamérica, con excepción de las zonas tropicales y subtropicales (Magnusson, 1954; Marbach, 2000), y se estima que el número de representantes en Chile sería cercano a las 60 especies (Galloway \& Quilhot, 1998).

\section{Conservación y los Cuernos del Desierto}

Uno de los registros más importantes asociados a este estudio es la presencia de Santessonia cervicornis (Follmann) Follmann o Cuernos del Desierto (Figura No 2) dentro del área concesionada al Centro del Desierto de Atacama. Esta especie se caracteriza por su morfología fruticosa, sin la presencia de estructuras de reproducción sexual y su hábito característicamente vagante, al no estar adherido a un sustrato establecido (Follmann, 1966).

Ya en el año 2001 (Muñoz-Schick et al., 2001) se indicó la presencia de esta especie en Alto Patache, y a partir de este se ha destacado al oasis como su única localidad conocida luego que Follmann (1995) la reportara extinta de su localidad tipo en el Morro Moreno, en la región de Antofagasta. Producto de la reducción de su área de distribución conocida, así como la existencia de una única población, es que esta especie ha sido clasificada como en Peligro Crítico de Extinción 
por el Consejo de Ministros para la Sustentabilidad y el Ministerio del Medio Ambiente de Chile en su XIII proceso de Clasificación de especies (MMA, 2017), de acuerdo al Reglamento de Clasificación de Especies de Chile (Decreto 29, MMA, 2012), convirtiéndola en una de las primeras especies de líquenes clasificadas en el país.

Figura No 2

Hábito de Santessonia cervicornis en Alto Patache

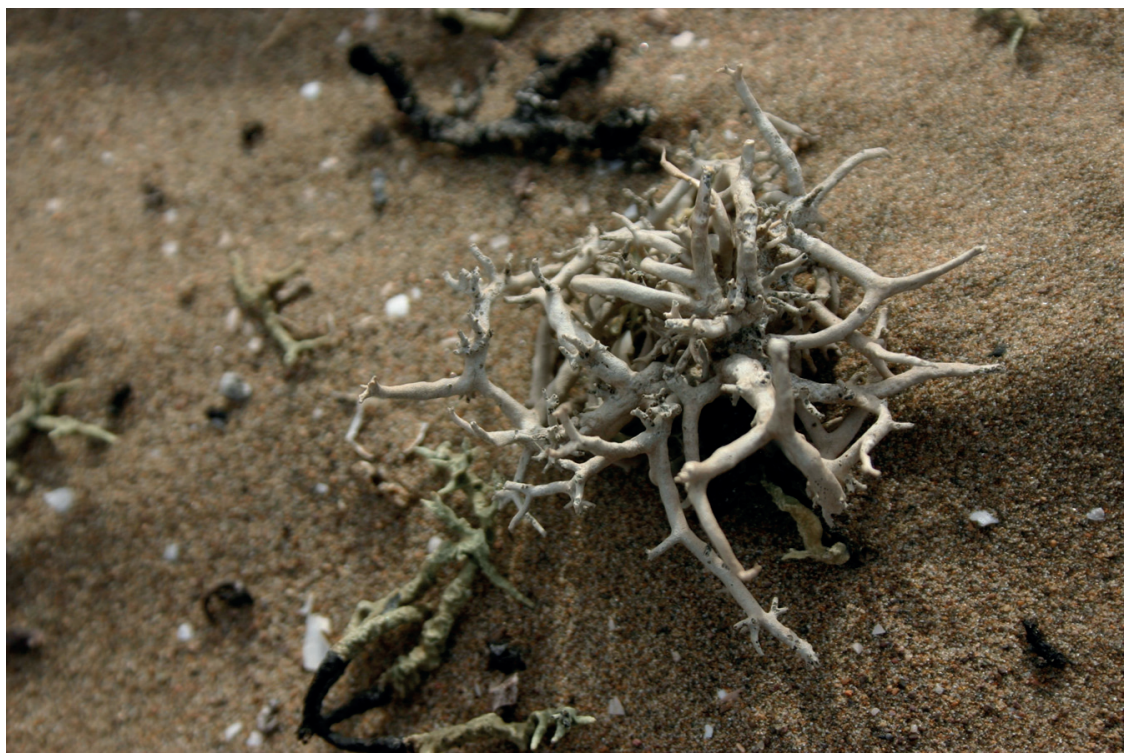

Fuente: Fotografía de Daniel Stanton.

\section{Consideraciones finales}

El oasis de neblina de Alto Patache revela ser un centro de diversidad liquénica de importancia nacional e internacional, considerando que, de 77 especies reportadas, 61 corresponden a nuevos registros para la región de Tarapacá, en tanto que 4 especies (Amandinea efflorescens, Diploicia canescens, Myriospora smaragdula y Rhizocarpon simillimum) corresponden a nuevos registros para Chile continental.

Se destaca que los registros reportados aquí solo representan una primera aproximación a la biota del sitio. No obstante lo anterior, ya indican una ampliación significativa del número de especies reconocidas para el área (Muñoz-Schick et al., 2001). Estos inicios auspiciosos, así como la diversidad liquénica reportada para otros oasis de neblina, como Cerro Moreno y Paposo, sugieren que revisiones más exhaustivas seguramente traerán adicionales registros junto con novedades taxonómicas, las que serán profundizadas en futuros estudios. Junto con esto, se destaca la presencia de la especie en Peligro Crítico Santessonia cervicornis, siendo Alto Patache su única localidad conocida. 
Finalmente, se promueve a Alto Patache como lugar idóneo para estudios de la ecología de líquenes. Esto considerando la gran variación en las distribuciones altitudinales, la que delata una gran gama de adaptaciones al ambiente hiperdesértico del Atacama, las que destacan aún más la particularidad biogeográfica de estos oasis de neblina (Kappen, 1982; Stanton, 2015).

\section{Referencias bibliográficas}

ARMESTO, J. \& CONTRERAS, L.C. Saxicolous lichen communities: nonequilibrium systems? American Naturalist, 1981, № 118, p. 597-604.

CABRERA, E.; HEPP, J.; GÓMEZ, M. \& CONTRERAS, S. Seed dormancy of Nolana jaffuelii I.M.Johnst. (Solanaceae) in the coastal Atacama Desert. Flora - Morphology, Distribution, Functional Ecology of Plants, 2015, No 214, p. 17-23.

DILLON, M.O.; ARANCIO, G. \& LUEBERT, F. Five new species of Nolana (Solanaceae-Nolaneae) from Chile. Arnaldoa, 2007, № 14, p. 191-212.

FEUERER, T. \& HAWKSWORTH, D.L. Biodiversity of lichens, including a world-wide analysis of checklist data based on Takhtajan's floristic regions. Biodiversity and Conservation, 2007, No 16, p. 85-98.

FEUERER, T. \& HÖHNE, H. Takhtajan's definition of global floristic regions revisited. Graphis Scripta, 2017, No 29, p. 40-43.

FOLLMANN, G. \& REDÓN, J. Erganzungen zur Flechtenflora der nordchilenischen Nebeloasen Fray Jorge und Talinay. Willdenowia, 1972, № 6, p. 431-460.

FOLLMANN, G. Eine felsbewohnende Flechtengesellschaft der mittel- und nordchilenischen Krustenformationen mit kennzeichnender Roccella portentosa (Mont.) Darb. Berichte der Deutschen Botanischen Gesellschaft, 1964, No 77, p. 262-274.

FOLLMANN, G. Fensterflechten in der Atacamawuste. Die Naturwissenschaften, 1965, No 52, p. 434-435.

FOLLMANN, G. Chilenische Wanderflechten. Berichte der Deutschen Botanischen Gesellschaft, 1966, No 79 , p. 453-462.

FOLLMANN, G. Die Flechtenflora der nordchilenischen Nebeloase Cerro Moreno. Nova Hedwigia, 1967, No 14 , p. 215-281.

FOLLMANN, G. Felsbewohnende Arthoniaceen der chilenischen Pazifikkuste. Willdenowia 1968, No 4, p. 365-382.

FOLLMANN, G. Darwin's "lichen oasis" above Iquique, Atacama Desert rediscovered. International Lichenological Newsletter, 1994, № 27, p. 23- 25. 
FOLLMANN, G. On the impoverishment of the lichen flora and the retrogression of the lichen vegetation in coastal central and northern Chile during the last decades. Cryptogamic Botany, 1995, No 5, p. 224-231.

FOLLMANN, G. An integrated key to and a critical survey of the South American representatives of the lichen family Roccellaceae (Arthoniales). Journal of the Hattori Botanical Laboratory, 2001, No 90, p. 251-267.

GALLOWAY, D.J. \& MARTICORENA, C. A bibliography of Chilean lichenology. Gayana Botánica, 1991, No 48, p. 17-66.

GALLOWAY, D.J. \& QUILHOT, W. Checklist of Chilean lichen-forming and lichenicolous fungi. Gayana Botánica, 1998, No 55, p. 111-185.

GALLOWAY, D.J. Lichens in Southern Hemisphere temperate rainforest and their role in maintenance of biodiversity. In: ALLSOPP, D.; COLWELL, R.R. \& HAWKSWORTH, D.L. (editors). Microbial Diversity and Ecosystem Function. Wallingford: CAB International, 1995, p. 125-135.

GALLOWAY, D.J. The lichens of Chile: present knowledge and future prospects. In: MARCELLI, M.P. \& SEAWARD, M.R.D. (editors). Lichenology in Latin America: History, Current Knowledge and Applications. Sao Paulo: CETESB - Companhia de Tecnologia de Saneamento Ambiental - Estado de Sao Paulo, 1998, p. 87-100.

GALLOWAY, D.J. Lichen biogeography. In: NASH, T.H. (editor). Lichen Biology. Cambridge: Cambridge University Press, Second Edition, 2008, p.315-335.

HIBBETT, D.S.; BINDER, M.; BISCHOFF, J.F.; BLACKWELL, M.; CANNON, P.F.; ERIKSSON, O.E.; HUHNDORF, S.; JAMES, T.; KIRK, P.M.; LÜCKING, R.; LUMBSCH, H.T.; LUTZONI, F.; MATHENY, P.B.; MCLAUGHLIN, D.J.; POWELL, M.J.; REDHEAD, S.; SCHOCH, C.L.; SPATAFORA, J.W.; STALPERS, J.A.; VILGALYS, R.; AIME, M.C.; APTROOT, A.; BAUER, R.; BEGEROW, R.; BENNY, G.L.; CASTLEBURY, L.A.; CROUS, P.W.; DAI, Y-C.; GAMS, W.; GEISER, D.M.; GRIFFITH, G.W.; GUEIDAN, C.; HAWKSWORTH, D.L.; HESTMARK, G.; HOSAKA, K.; HUMBER, R.A.; HYDE, K.D.; IRONSIDE, J.E.; KÕLJALG, U.; KURTZMAN, C.P.; LARSSON, K-H.; LICHTWARDT, R.; LONGCORE, J.; MIADLIKOWSKA, J.; MILLER, A.; MONCALVO, J-M.; MOZLEY-STANDRIDGE, S.; OBERWINKLER, F.; PARMASTO, E.; REEB, V.; ROGERS, J.D.; ROUX, C.; RYVARDEN, L.; SAMPAIO, J.P.; SCHÜßLER, A.; SUGIYAMA, J.; THORN, R.G.; TIBELL, L.; UNTEREINER, W.A.; WALKER, C.; WANG, Z.; WEIR, A.; WEISS, M., WHITE, M.M.; WINKA, K.; YAO, Y-J. \& ZHANG, N. A higher-level phylogenetic classification of the Fungi. Mycological Research, 2007, No 111, p. 509-547.

JAKLITSCH, W.; BARAL, H-O.; LÜCKING, R.; LUMBSCH, H.T. \& FREY, W. Syllabus of Plant Families - A. Engler's Syllabus der Pflanzenfamilien Part 1/2: Ascomycota. Stuttgart: Borntraeger Verlagsbuchhandlung, 13 edition, 2016.

JAMES, T.Y.; KAUFF, F.; SCHOCH, C.; MATHENY, P.B.; HOFSTETTER, V.; COX, C.J.; CELIO, G.; GUEIDAN, C.; FRAKER, E.; MIADLIKOWSKA, J.; LUMBSCH, H.T.; RAUHUT, A.; REEB, V.; ARNOLD, A.E.; AMTOFT, A.; STAJICH, J.E.; HOSAKA, K.; SUNG, G.-H.; JOHNSON, D.; O'ROURKE, B.; CROCKETT, M.; BINDER, M.; CURTIS, J.M.; SLOT, J.C.; WANG, Z.; WILSON, A.W.; SCHÜßLER, A.; LONGCORE, J.E.; O'DONNE- 
LL, K.; MOZLEY-STANDRIDGE, S.; PORTER, D.; LETCHER, P.M.; POWELL, M.J.; TAYLOR, J.W.; WHITE, M.M.; GRIFFITH, G.W.; DAVIES, D.D.; HUMBER, R.A.; MORTON, J.B.; SUGIYAMA, J.; ROSSMAN, A.; ROGERS, J.D.; PFISTER, D.H.; HEWITT, D.; HANSEN, K.; HAMBLETON, S.; SHOEMAKER, R.A.; KOHLMEYER, J.; VOLKMANN-KOHLMEYER, B.; SPOTTS, R. A.; SERDANI, M.; CROUS, P.W.; HUGHES, K.W.; MATSURA, K.; LANGER, E.; LANFER, G.; UNTEREINER, W.A.; LÜCKING, R.; BÜDEL, B.; GEISER, D.M.; APTROOT, A.; DIEDERICH, P.; SCHMITT, I.; SCHULTZ, M.; YAHR, R.; HIBBETT, D.S.; LUTZONI, F.; MCLAUGHLIN, D.J.; SPATAFORA, J.W. \& VILGALYS, R. Reconstructing the early evolution of fungi using a six-gene phylogeny. Nature, 2006, $N^{\circ} 443$, p. 818-822,

KAPPEN, L. Lichen oases in hot and cold deserts. Journal of the Hattori Botanical Laboratory, 1982, $N^{\circ} 53$, p. 325-330.

LANGE, O.L. \& REDÓN, J. Epiphytische Flechten im Bereich einer chilenischen "Nebeloase" (Fray Jorge). II. Okophysiologische Charakterisierung von CO2- Gaswechsel und Wasserhaushalt. Flora, $1982, N^{\circ} 174$, p. $245-284$.

LANGE, O.L.; GREEN, T.G.A.; MELZER, B.; MEYER, A. \& ZELLNER, H. Water relations and CO2 exchange of the terrestrial lichen Teloschistes capensis in the Namib fog desert: measurements during two seasons in the field and under controlled conditions. Flora, 2006, № 201, p.268-280.

LARRAíN, H.; VELÁSQUEZ F.; LÁZARO, P.; CERECEDA, P.; OSSES, P. \& PÉREZ. L. Un yacimiento de cazadores recolectores marinos en la terraza litoral de Bajo Patache, sur de lquique. POLIS, 2004, $N^{\circ}$ 7. Disponible en Internet: http://polis.revues.org/6320

LEMBCKE, H. \& WEISSER, P.J. The distribution of the genera of Chilean Cactaceae. Aloe 1979, Nº 17 , p. $9-26$

LÜCKING, R.; WIRTH, V.; FERRARO, L. \& CÁCERES, M.E.S. Foliicolous lichens from Valdivian temperate rain forest of Chile and Argentina: evidence of an austral element, with description of seven new taxa. Global Ecology and Biogeography, 2003, N¹2, p. 21-36.

LÜCKING, R.; HODKINSON, B.P. \& LEAVITT, S.D. The 2016 classification of lichenized fungi in the Ascomycota and Basidiomycota - Approaching one thousand genera. The Bryologist, 2017, $N^{\circ} 119$, p. 361-416.

LUEBERT, F. \& PLISCOFF, P. Sinopsis bioclimática y vegetacional de Chile. Santiago de Chile: Editorial Universitaria, 2006.

LUMBSCH, H.T. \& HUHNDORF, S. Myconet Volume 14. Part One. Outline of Ascomycota - 2009. Part Two. Notes on Ascomycete Systematics. $N^{\circ}$ 4751-5113. Fieldiana Life and Earth Sciences 2010, No 1, p. 1-64.

MAGNUSSON, A.H. Key to saxicolous Buellia species, mainly from South America. Arkiv för Botanik, 1954, Nº 3, p. 205-221.

MARBACH, B. Corticole und lignicole Arten der Flechtengattung Buellia sensu lato in den Subtropen und Tropen. Bibliotheca Lichenologica, 2000, N 74, p. 1-384. 
MINISTERIO DEL MEDIO AMBIENTE. Decreto $n^{\circ} 29$, Aprueba reglamento para la clasificación de especies silvestres según estado de conservación. Santiago de Chile: Ministerio del Medio Ambiente, 2012.

MINISTERIO DEL MEDIO AMBIENTE. Decreto Supremo $n^{\circ}$ 6, aprueba y oficializa clasificación de especies según estado de conservación, Décimo Tercer proceso. Santiago de Chile: Ministerio del Medio Ambiente, 2017.

MOREIRA-MUÑOZ, A. Plant Geography of Chile. Plant and Vegetation 5. Amsterdam: Springer Netherlands, 2011.

MUÑOZ-SCHICK, M.; PINTO, R.; MESA, A. \& MOREIRA-MUÑOZ, A. Oasis de neblina en los cerros costeros del sur de lquique, región de Tarapacá, Chile, durante el evento El Niño 1997-1998. Revista Chilena de Historia Natural, 2001, № 74, p. 389-405.

OSSA, P.G.; PÉREZ, F. \& ARMESTO, J.J. Phylogeography of two closely related species of Nolana from the coastal Atacama Desert of Chile: post-glacial population expansions in response to climate fluctuations. Journal of Biogeography, 2013, № 40, p. 2191-2203.

ØVSTEDAL, D.O. \& LEWIS-SMITH, R.I. Lichens of Antarctica and South Georgia: A Guide to Their Identification and Ecology. Cambridge: Studies in Polar Research, Cambridge University Press, 2001.

PEREIRA, I. \& SAN MARTIN, J. Corticolous lichen flora of a relict forest of Nothofagus alessandrii in central Chile. Cryptogamie, Bryologie-Lichénologie, 1998, N¹9, p. 59-72.

PEREIRA, I.; SAN MARTíN, J. \& ROUX, C. Patrón florístico de líquenes epifitos de Nothofagus glauca in un bosque costero de Chile central. Gayana Botánica, 1999, № 56, p. 69-76.

PEREIRA, I.; SAN MARTÍN, J. \& MOYA, M. Epiphytic lichens on Gomortega keule (Gomortegaceae) in the coastal mountains of central Chile. Mitteilungen aus dem Institut für Allgemeine Botanik in Hamburg, 2002, N³0-32, p. 171-185.

PEREIRA, I.; MÜLLER, F. \& VALDERRAMA, A. Diversity and distribution of bryophytes and lichens of El Colorado, Central Chile. Nova Hedwigia, 2006, № 83, p. 117-127.

PEREIRA, I., MÜLLER, F. \& MOYA, M. Influence of Nothofagus bark $\mathrm{pH}$ on the lichen and bryophytes richness, Central Chile. Gayana Botánica, 2014, № 71, p. 120-130.

PEREIRA, I.; WANG, X.Y.; OH, S-O.; SÁNCHEZ, P. \& HUR, J-S. Lichens of the surrounding areas of Termas of Chillán and Las Trancas, Bío-Bío Region, Chile. Gayana Botánica, 2016, № 73, p. 104-112.

PHILLIPPI, F. A visit to the northernmost forest of Chile. Journal of Botany, 1884, N²2, p. 202-211

PHILIPPI, F. Reise nach der Provinz Tarapacá. Verhandlungen des Deutschen Wissenschaftlichen Vereins in Santiago, 1886, N 1, p. 135-163. 
PINTO, R. \& LUEBERT, F. Datos sobre la flora vascular del desierto costero de Arica y Tarapacá, Chile, y sus relaciones fitogeográficas con el Sur de Perú. Gayana Botánica, 2009, № 66, p. 28-49

QUILHOT, W.; PEREIRA, I.; GUZMÁN, G.; RODRÍGUEZ, R. \& SEREY, I. Categorías de conservación de líquenes nativos de Chile. Boletín del Museo Nacional de Historia Natural, 1998, № 47, p. 9-22.

QUILHOT, W.; CUELLAR, M.; DÍAZ, R.; RIQUELME, F. \& RUBIO, C. Lichens of Aisen, Southern Chile. Gayana Botánica, 2012, №69, p. 57-87.

REDÓN, J. \& QUILHOT, W. Los líquenes de las Islas de Juan Fernández I: estudio preliminar. Anales del Museo de Historia Natural, Valparaíso, 1977, N 10, p. 15-26.

REDÓN, J. \& WALKOWIAK, A. Estudio Preliminar de la Flora liquénica del Parque Nacional La Campana. I: Resultados sistemáticos. Anales el museo de Historia Natural, Valparaíso, 1978, № 11, p. 19-36.

REDÓN, J. Beobachtungen zur Geographie und Okologie der chilenischen Flechtenflora. Journal of the Hattori Botanical Laboratory, 1973, N³7, p. 153-167.

RUNDEL, P.W. \& DILLON, M. Ecological patterns in the Bromeliaceae of the lomas formations of Coastal Chile and Peru. Plant Systematics and Evolution, 1998, № 212, p. 261-278.

RUNDEL, P.W. Ecological relationships of desert fog zone lichens. The Bryologist, 1978, N 81, p. 277-293.

RUNDEL, P.W. Corticolous lichen communities of Nothofagus dombeyi on Volcan Villarica in southern Chile. The Bryologist, 1980, № 83, p. 82-84.

RUNDEL, P.W. The role of morphology in the water relations of desert lichens. Journal of the Hattori Botanical Laboratory, 1982, N 53, p. 315-320.

SCHOCH, C.; SUNG, G.-H.; LÓPEZ-GIRÁLDEZ, F.; TOWNSEND, J.P.; MIADLIKOWSKA, J.; HOFSTETTER, V.; ROBBERTSE, B.; MATHENY, B.; KAUFF, F.; WANG, Z.; GUEIDAN, C.; ANDRIE, R.M.; TRIPPE, K.; CIUFETTI, L.M.; WYNNS, A.; FRAKER, E.; HODKINSON, B.P.; BONITO, G.; GROENEWALD, J.Z.; ARZANLOU, M.; DE HOOG, G.S.; CROUS, P.W.; HEWITT, D.; PFISTER, D.; PETERSON, K.; GRYZENHOUT, M.; WINGFIELD, M.J.; APTROOT, A.; SUH, S.-O.; BLACKWELL, M.; HILLIS, D.M.; GRIFFITH, G.W.; CASTLEBURY, L.A.; ROSSMAN, A.; LUMBSCH, H.T.; LÜCKING, R.; BÜDEL, B.; RAUHUT, A.; DIEDERICH, P.; ERTZ, D.; GEISER, D.M.; HOSAKA, K.; INDERBITZIN, P.; KOHLMEYER, J.; VOLKMANN-KOHLMEYER, B.; MOSTERT, L.; O'DONNELL, K.; SIPMAN, H.; ROGERS, J.D.; SHOEMAKER, R.; SUGIYAMA, J.; SUMMERBELL, R.C.; UNTEREINER, W.; JOHNSTON, P.R.; STENROOS, S.; ZUCCARO, A.; DYER, P.S.; CRITTENDEN, P.D.; COLE, M. S.; HANSEN, K.; TRAPPE, J.M.; YAHR, R.; LUTZONI, F. \& SAPATAFORA, J.W. The Ascomycota tree of life: a phylum-wide phylogeny clarifies the origin and evolution of fundamental reproductive and ecological traits. Systematic Biology, 2009, № 58, p. 224-239.

SCHULZ, N. Loma-Formationen der Küsten-Atacama/Nordchile unter besonderer Berücksichtigung rezenter Vegetations- und Klimaveränderungen. Erlangen: PhD thesis, Geographical Institute, Erlangen-Nürnberg University, 2006. 
STANTON, D. Small scale fog-gradients change epiphytic lichen shape and distribution. The Bryologist, 2015, N 118, p. 241-244.

TAKHTAJAN, A. Floristic regions of the world. Los Angeles: University of California Press, 1986.

TEHLER, A.; IRESTEDT, M.; WEDIN, M. \& ERTZ, D. Origin, evolution and taxonomy of American Roccella (Roccellaceae, Ascomycetes). Systematics and Biodiversity, 2009, N 7, p. 307-317.

TU, T.; DILLON, M.O.; SUN, H. \& WEN, J. Phylogeny of Nolana (Solanaceae) of the Atacama and Peruvian deserts inferred from sequences of four plastid markers and the nuclear LEAFY second intron. Molecular Phylogenetics and Evolution, 2008, № 49, p. 561-573.

VARGAS, R. \& BECK, A. Photobiont selectivity and specificity in Caloplaca species in a fog-induced community in the Atacama Desert, northern Chile. Fungal Biology, 2012, No 116, p. 665-676.

VARGAS, R. \& MORANO, C. Hongos liquenizados en morrenas del monte San Lorenzo, Región de Aisén, Chile. Gayana Botánica, 2014, № 71, p. 140-146.

ZAHLBRUCKNER, A. Die Flechten der Juan-Fernandez-Inseln. In: Skottsberg, C. (editor). The natural history of Juan Fernandez and Easter Island. II. Uppsala: Almqvist \& Wiksells Boktryckeri, 1924.

ZULOAGA, F.O.; MORRONE, O. \& BELGRANO, M.J. (editores). Catálogo de las Plantas Vasculares del Cono Sur: Argentina, Sur de Brasil, Chile, Paraguay y Uruguay. St. Louis, MO: Missouri Botanical Garden Press, 2008, № 107, p. 1715-1830. 